\title{
COMMENTS ON PROFESSOR LLIBOUTRY'S PAPER
}

\author{
By J. F. Nye \\ (University of Bristol)
}

In so far as Professor Lliboutry is trying to make the theory of glacier flow more realistic one can only wish him well and hope that he is on the right track. Leaving aside for the moment the question of the crevasses, my objections were entirely directed against his calculation made with a specific model, namely a perfectly plastic glacier, and here the questions are not ones of opinion, but of the truth or falsity of mathematical deductions.

As a first approximation Lliboutry's formulae for the glacier profile and the stresses are, as I submitted before, correct but not new, and on this we seem to be in agreement. The difficulties begin when we come to the second approximation. I said that the method Lliboutry used appeared to me to be completely fallacious. I did not say why this was so because it seemed sufficient to point out that the resulting "solution" did not satisfy one of the boundary conditions: that the upper surface of the glacier should be free from shear stress. Lliboutry now says that the figure (Fig. 5 of the original paper) which makes this obvious should not have been drawn and insists that the second approximation for the profile remains unchallenged. It is therefore now necessary for me to say, as briefly as I can, why the method used is fallacious.

It is a question of the correct way of manipulating approximate equations. Since the mistake is present whether the slope of the bed $\beta$ is zero or not, I shall take the case $\beta=0$ for simplicity. The equation after (12) on p. 255 of the original paper is

which for $\beta=0$ reduces to

$$
\tau=\rho g(e-z) \tan \alpha \cdot \cos \beta \text {, }
$$

$$
\tau=\rho g(e-z) \tan \alpha .
$$

We seem to be agreed that this is only a first approximation, in the sense that it approaches the true equation for $\tau$ as $\alpha$ approaches zero (it certainly cannot be true for large $\alpha$ ). The true equation for $\tau$ must therefore be

$$
\tau=\rho g e\left\{\left(1-\frac{z}{e}\right) \alpha+O\left(\alpha^{2}\right)\right\},
$$

where $O\left(\alpha^{2}\right)$ means terms of order $\alpha^{2}$ and higher, which will, in the absence of proof to the contrary, be functions of $x$ and $z$. If Lliboutry thinks the terms he omits are of order $\alpha^{3}$ or higher rather than $\alpha^{2}$, it is up to him to say so explicitly and to prove it.

On the bed, $z=0$ and $\tau=\tau_{\circ}$; whence

$$
\tau_{\circ}=\rho g e\left\{\alpha+O\left(\alpha^{2}\right)\right\}
$$

the terms $O\left(\alpha^{2}\right)$ being functions of $x$. Then, dividing (I) by (2), we find

$$
\tau=\tau_{\circ}\left\{\left(1-\frac{z}{e}\right)+O(\alpha)\right\}
$$

as the full version of Lliboutry's equation (1 3$)$. The upper surface $(z=e)$ must be free from shear traction, but since the surface is not perpendicular to the $z$ axis, which is vertical, $\tau$ will not in general be zero at $z=e$. Thus we may notice that the terms $\tau_{0} O(\alpha)$ in equation (3) are essential for the satisfying of the upper boundary condition.

It then follows that Lliboutry's equation (I4) omits terms of the form $\tau_{\circ} O(\alpha)$. In order to substitute in the second equation of equilibrium, which is (with $\beta=0$ )

$$
\frac{\partial \sigma_{z}}{\partial z}+\frac{\partial \tau}{\partial x}=-\rho g
$$


we first differentiate the above equation (3) and obtain

$$
\frac{\partial \tau}{\partial x}=\tau_{\circ} z \frac{e^{\prime}}{e^{2}}+\text { terms arising from the differentiation of the small terms } \tau_{0} O(\alpha) .
$$

We do not know how these terms $\tau_{\circ} O(\alpha)$ depend on $x$, but when they are differentiated there is no reason why they should be smaller than $\left(\tau_{\circ} / a\right) O(\alpha)$, where $a$ is some length associated with the $x$ direction. Again, Lliboutry may think they are of higher order than this, but if so he must say so explicitly and prove it. Substituting in (4) we have

Now, since $\beta=0$,

$$
\frac{\partial \sigma_{z}}{\partial z}=-\rho g-\tau_{\circ} z \frac{e^{\prime}}{e^{2}}+\frac{\tau_{\circ}}{a} O(\alpha) .
$$

$$
e^{\prime}=\frac{d e}{d x}=\tan \alpha,
$$

and so the second term on the right in the above equation $(5)$ is of order $\left(\tau_{0} / e\right) \alpha$, which is comparable in magnitude to the terms indicated by $\left(\tau_{\circ} / a\right) O(\alpha)$ (unless it can be shown that $a \gg e$, which Lliboutry has not done). Thus the term $-\tau_{\circ} z\left(e^{\prime} \mid e^{2}\right)$ which Lliboutry calculates and which leads him to his second approximation for the profile is of the same order as the terms which are omitted. It is therefore quite valueless.

Lliboutry thus goes on to omit terms of order $\left(\tau_{0} z / a\right) O(\alpha)$ in the expression for $\sigma_{z}$ (sixth equation on p. 256). He then puts in the boundary condition $\sigma_{z}=H$ at $z=e$, which is only approximately correct. The correct boundary condition is that the normal stress on the surface at $z=e$ is $H$. Since the surface is not perpendicular to the $z$ axis $\sigma_{z}$ will not be exactly equal to $H$. It is in fact equal to $H\left\{\mathrm{I}+O\left(\alpha^{2}\right)\right\}$. The result is that Lliboutry's equation ( $I_{5}$ ) for $\sigma_{z}$, which he repeats in his reply as his sixth equation, omits terms of order $\left(\tau_{\circ} z / a\right) O(\alpha)$ which are comparable with the term $\frac{\tau_{\circ}}{2} e^{\prime}\left(\mathrm{I}-\frac{z^{2}}{e^{2}}\right)$ which he retains. His equation (I5) for $\sigma_{x}$, repeated in his reply as his seventh equation, omits not only these terms $\left(\tau_{\circ} z / a\right) O(\alpha)$ but also terms $\tau_{\circ} O(\alpha)$ which come from the full version of his equation (I4). Thus, even if it could be shown that $a \gg e$, the analysis would still fail. (I do not understand why he credits me with these false equations for $\sigma_{z}$ and $\sigma_{x}$, and with the idea that $\sigma_{x}$ at the surface is $H \sin (\alpha-\beta)$. I cannot recollect having published such ideas and should be glad to know the exact references.) The second approximation to the profile is based on this erroneous equation (I5) for $\sigma_{x}$ and accordingly fails. This having been said, the question about the slope at the origin no longer arises.

It may well be that a solution to the problem of the glacier profile may be obtained by carrying out numerically a step-by-step extension of the cycloidal slip-line field which we already know, by using the standard methods in the theory of the plane-strain slip-line field which are fully described in Hill's book and elsewhere. If this were done I conjecture that the solution would terminate along a limiting slip line rather as shown in Fig. $7 \mathrm{~b}$ of Lliboutry's original paper, except that the velocity need not be parallel to the limiting slip line. The wedge-shaped mass ahead would be pushed forward rigidly. As fast as it was melted away on its surface it would be fed with new material flowing across the limiting slip line. That is conjecture. On the other hand we can say something about the slope of a glacier at its snout which is independent of all flow theories and is simply geometry. Namely, that in a glacier front which is not advancing or retreating the slope $\theta$ of the front relative to the bed is given by

$$
\sin \theta=\frac{r}{u}
$$

where $r$ is the rate of ablation measured perpendicular to the surface and $u$ is the forward velocity parallel to the bed, both measured at the snout. 
Lliboutry says I accuse him of not taking account of ablation when computing his velocity solution. In fact I accused him of not taking account of ablation when calculating his profile. What I wrote was "Furthermore, it is said in the paper that one can calculate the profile entirely from the stress solution and without reference to the distribution of velocities (bottom of p. 264). But surely the profile must be determined to some extent by the distribution of ablation." I adhere to this view.

Crevasse formation. I agree that a place where the tensile strength is zero would be a crack. The point I should like to take up is whether, given a crack, one needs a stress or a strain to open it up. I say a stress; Lliboutry says a strain. The simplest case which brings out the difference between the two views is shown in Figure $\mathrm{I}$ (below). In Fig. ra and b a plastic
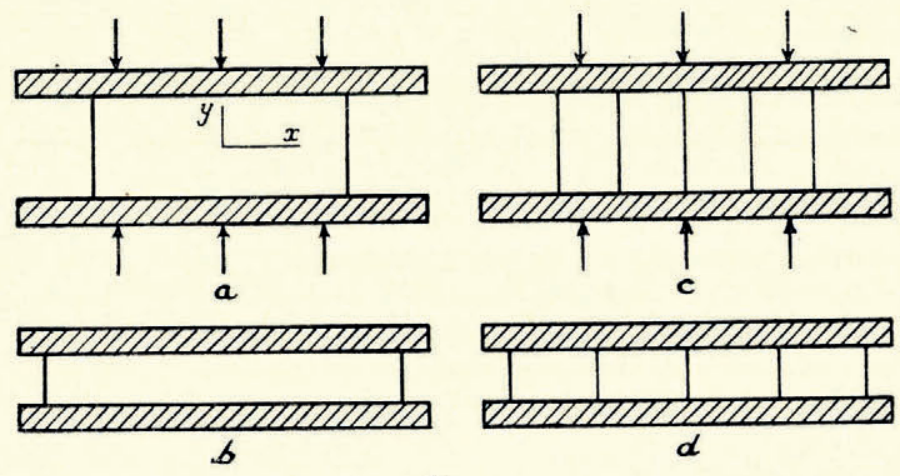

Fig. $I$

block is compressed between two completely smooth parallel plates. (The relation to a glacier is that the plates represent the valley sides.) If the block is homogeneous and has no cracks, the strain is homogeneous and the material is squeezed out sideways. $\partial u / \partial x$ is positive; but $\sigma_{x}$ is zero. There is thus a strain in the $x$ direction but no stress. The question is: if we now place cracks perpendicular to $O x$, will they open or not? I say no; Lliboutry presumably says yes.

Suppose now that the original block is made up of a number of smaller blocks as shown in Fig. Ic, with no cohesion between them. When the composite block is compressed they will all strain homogeneously just as the big one did (Fig. Id). The outer ones will of course slide outwards to make room for the increased width of the inner ones, but since the plates are smooth this introduces no extra forces. The essential point is that the surfaces separating the blocks remain flat; they do not bow inwards and leave cavities between the blocks. The surfaces separating the blocks are simply very large cracks and they do not open even though there is a tensile strain parallel to $O x$. If, however, we apply a tensile stress along $O x$ the whole thing comes apart.

I have discussed the block between parallel plates rather than the strip passing between rolls because the strains and stresses in the block are homogeneous and it brings out the essential point. The rolling case involves inhomogeneous stresses and is therefore more complicated in detail.

May I end by repeating what I said at the beginning: that I am entirely in favour of trying to make the solution to a theoretical problem applicable to real life-that is what physics is about. But, at the same time, let us be sure that the theoretical problem has been adequately solved. In the present case Lliboutry has put forward what he says is a solution, but he has not justified it. 